Cinémas

Revue d'études cinématographiques

Journal of Film Studies

\title{
La critique et le public
}

\section{Yannick Mouren}

Volume 6, numéro 2-3, printemps 1996

\section{La Critique cinématographique}

URI : https://id.erudit.org/iderudit/1000972ar

DOI : https://doi.org/10.7202/1000972ar

Aller au sommaire du numéro

Éditeur(s)

Cinémas

\section{ISSN}

1181-6945 (imprimé)

1705-6500 (numérique)

Découvrir la revue

Citer cet article

Mouren, Y. (1996). La critique et le public. Cinémas, 6(2-3), 65-79.

https://doi.org/10.7202/1000972ar

\section{Résumé de l'article}

Une question hante les agents du champ cinématographique: « Le public a-t-il raison? " Le discours critique évite généralement de se positionner par rapport à cette interrogation. Sa présence, toujours sous-jacente, peut être mise en lumière en analysant diverses recensions du film Les Visiteurs, le plus grand succès français de ces dernières années. On peut expliquer les différentes prises de position sur ce film (du dithyrambe sans réticences au démolissage expéditif) si l'on tient compte de plusieurs variables comme la date de parution de l'article (impliquant ignorance ou connaissance du triomphe du film) et les nombreuses positions critiques adoptées dans le champ. 


\section{La critique et le public}

\section{Yannick Mouren}

\section{RÉSUMÉ}

Une question hante les agents du champ cinématographique: "Le public a-t-il raison?» Le discours critique évite généralement de se positionner par rapport à cette interrogation. Sa présence, toujours sous-jacente, peut être mise en lumière en analysant diverses recensions du film Les Visiteurs, le plus grand succès français de ces dernières années. On peut expliquer les différentes prises de position sur ce film (du dithyrambe sans réticences au démolissage expéditif) si l'on tient compte de plusieurs variables comme la date de parution de l'article (impliquant ignorance ou connaissance du triomphe du film) et les nombreuses positions critiques adoptées dans le champ.

\section{ABSTRACT}

One question haunts those who work in the cinematographic field: "Is the public right?" Normally criticism avoids taking a stand on this issue. Its unacknowledged relevance may be illuminated by analyzing various responses to the film Les Visiteurs, the biggest French box-office success of recent years. One can explain the various stands taken regarding the film (from unabashed hymn of praise to hatchet job) by looking at variables like the article's publication date (implying awareness or ignorance of the triumph of the film), and the critical position taken towards it.

Il est bon, au moins pour la clarté de l'exposé, de présenter d'emblée le postulat sur lequel le présent travail sera construit. Ce postulat est directement emprunté aux travaux du sociologue Pierre Bourdieu. Le cinéma sera donc, ici, considéré comme un champ ' artistique, au même titre que le champ littéraire, ou le 
champ pictural. Les différents champs artistiques présentent des différences et des ressemblances, que l'on appellera les "lois générales des champs" (Bourdieu, 1984, p. 113). La loi générale de fonctionnement la plus importante des champs artistiques peut être ainsi décrite: deux logiques y coexistent et sont en lutte constante: celle qui pousse les agents à amasser et à garder le plus possible de capital spécifique (reconnaissance des pairs, prestige, consécration institutionnalisée ou non) et celle qui pousse les agents à gagner les profits financiers les plus élevés. Une particularité, cruciale, dans ce rapport: les gagnants d'une catégorie (capital spécifique) sont souvent les perdants de l'autre catégorie (capital économique) et vice versa.

Le septième art présente une caractéristique qui le distingue radicalement des autres champs artistiques: c'est le seul champ (avec l'architecture) qui nécessite, pour la production d'une œuvre, des capitaux très importants (le coût moyen d'un film français était de 24 millions de francs en 1994 et le film de référence de la présente étude a coûté 60 millions de francs en 1993). L'évidence, sans cesse énoncée, ne peut que s'imposer : la fabrication de l'image cinématographique est un processus coûteux. Il s'ensuit que l'emprise de l'argent ${ }^{2}$ dans le champ cinématographique est considérable sans pour autant faire de ce champ un commerce comme un autre.

Pour mettre en lumière la coexistence de la logique spécifique et de la logique économique dans le champ cinématographique, il est possible de prendre comme angle d'attaque ce sous-champ du champ cinématographique que consitue la critique. Et l'on ne s'étonnera pas de constater que la critique présente les mêmes propriétés que le champ cinématographique dans son entier; c'est-à-dire que certains de ses représentants qui sont situés le plus près du pôle économique sont particulièrement sensibles au verdict du public (le fameux "nombre d'entrées"); à l'inverse, ses représentants qui sont situés le plus près du pôle spécifique s'efforcent d'évaluer le film en faisant abstraction de la réponse du public à l'offre qui lui est faite.

La lecture des discours des divers agents engagés dans le champ cinématographique (réalisateurs, producteurs, critiques) montre qu'une question les hante: "Le public a-t-il raison?" 
Répondre de manière positive, c'est faire découler le jugement de valeur que l'on porte sur un film du succès ou de l'insuccès du film en question. Ceux qui sont inféodés au verdict du public sont les agents les plus proches du pôle économique, en gros, les producteurs et réalisateurs de productions cinématographiques que l'on dénommait il y a quelques années "commerciaux" et que l'on désigne maintenant par l'expression "grand public".

Mais la critique de cinéma, qui se fixe comme mission essentielle d'évaluer les films qui sortent, autrement dit de séparer le bon grain de l'ivraie dans la moisson cinématographique, bénéficie d'une situation lui permettant de n'avoir pas à se positionner directement par rapport à cette question de la justesse des jugements du public. Elle voit les longs métrages soumis à son jugement dans des projections de presse, avant qu'ils ne soient sur les écrans. Elle ne peut donc pas savoir quelle sera la sanction du public et les analyses, aussi fines et pertinentes soientelles, que feront les exégètes ne présagent pas, et ne présageront jamais, de façon infaillible les réactions des spectateurs. Certes, la critique revient assez régulièrement sur les œuvres dont elle vient de parler, par exemple à l'occasion des bilans de début d'année avec les fameuses «listes des 10 meilleurs films de l'année», pratiquées par plus d'une revue spécialisée. Et comme la liste des 10 plus grands succès de l'année est à la disposition de tous, la critique ne peut échapper à la comparaison. Pourtant, sauf erreur de ma part, elle préfere soit donner une liste ${ }^{3}$ sans la commenter (c'est le cas de la revue Positif), soit publier d'abord la liste de la rédaction, et le mois suivant la liste émanant du lectorat (ce que font Les Cabiers du cinéma, par exemple).

Par chance pour celui qui veut se livrer à une socio-analyse du discours critique, en 1993, le 27 janvier, est sorti sur les écrans un film français de Jean-Marie Poiré, Les Visiteurs, qui a connu un succès fulgurant (il a échappé à la règle qui veut que le succès décroît, du moins à partir de la troisième semaine, d'une semaine sur l'autre). Aussi, lorsqu'on étudie l'ensemble des critiques parues à propos de cette production, la traditionnelle opposition entre les revues spécialisées de cinéma et les autres journaux (les hebdomadaires généralistes comme L'Express, Le Point... et les quotidiens) n'est plus pertinente. C'est pourquoi 


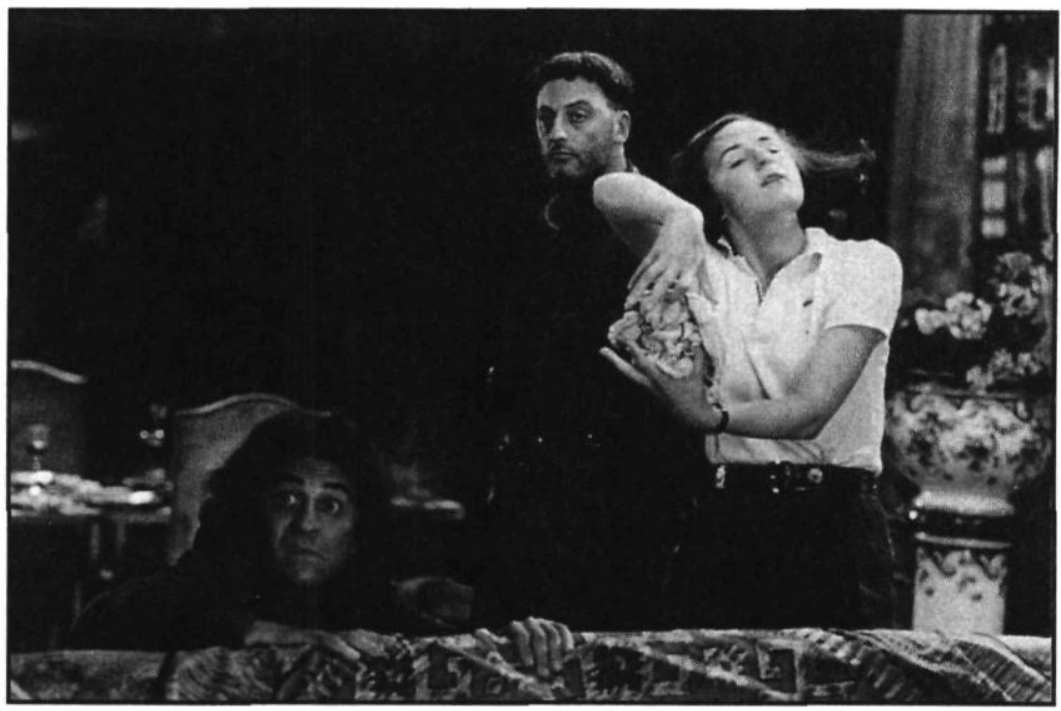

\section{Les Visifeurs de Jean-Marie Poiré (1 993)}

le critère qui va être pris en compte est la date à laquelle la critique a été écrite. Les recensions retenues vont donc être réparties en trois sections : la première sera consacrée aux articles concomitants de (ou précédant) la sortie du film, écrits dans l'ignorance de son succès, la seconde aux articles publiés une ou deux décades après sa sortie (essentiellement dans les hebdomadaires), et la troisième aux articles parus dans les revues de cinéma comme Positifet Les Cahiers du cinéma au mois de mars, c'est-à-dire plus d'un mois après sa sortie, alors que son succès phénoménal était connu de tous.

\section{Les articles écrits dans l'ignorance de son succès}

Les Visiteurs avaient fait l'objet d'une projection ouverte à toute la presse juste la veille de sa sortie. Une autre projection de presse avait eu lieu plusieurs semaines auparavant à laquelle n'avaient été invités que certains journalistes: c'est une pratique, déplorée par ses victimes mais de plus en plus courante qui permet d'écarter des projections de presse ceux dont une recension précédente n'a pas eu l'heur de plaire aux puissances invitantes. Les "papiers" sur cette comédie ont donc été publiés le 27 janvier dans les quotidiens "chanceux" (invités à la première pro- 
jection), le 28 ou le 29 pour les autres. À la lecture de ces critiques, on s'aperçoit que, dans la mesure où le journaliste s'est risqué à laisser passer sur son traitement de texte des termes pouvant être considérés comme des jugements de valeur, c'est une espèce de tiédeur, légèrement défavorable, qui prévaut. Encore faut-il prendre des précautions quand on lance de telles affirmations, tant les critiques des grands quotidiens sont passés maîtres dans l'art de déguiser une promotion de film en une critique "objective" ayant toutes les apparences du texte informatif. Cette prétendue neutralité n'est en fait qu'un leurre, parce qu'il est évident que le jugement de valeur est implicite dans la taille de l'espace-papier (texte + photo) accordé au film. Une demi-page "neutre", dans Le Figaro, consacrée à un film bénéficiant d'une campagne publicitaire importante et/ou d'un battage médiatique aura, sans doute, plus d'effet (si tant est que le critique en ait), que 50 lignes laudatives (mais reléguées dans un coin de page) consacrées à un film sans budget publicitaire. Cela est confirmé par la lecture des enquêtes de motivation des spectateurs de cinéma : à la question "Pourquoi avez-vous choisi de voir tel film?", il est très souvent répondu "Parce qu'on en a beaucoup parlé dans les médias». Tout laisse à penser que ce "beaucoup" est produit par des variables telles que la mention du film à la télévision ou la taille des articles dans les quotidiens et les hebdomadaires.

"Tiédeur légèrement défavorable", donc, chez les critiques de quotidiens, du moins chez ceux qui se refusent à se cantonner dans ces "papiers" qui se veulent purement informationnels. C'est ainsi que dans Le Parisien libéré, quotidien dont on s'attendrait à ce qu'il cherche à se trouver le plus près possible des goûts du plus grand nombre, le critique annonce, dès sa première phrase, ses réticences: "On n’a jamais vu le cinéma français investir autant d'argent dans un film qui n'est somme toute qu'une grosse farce ${ }^{4}$." Et dans les derniers paragraphes, qui sont consacrés à l'exposition de ce qui s'apparente à des jugements de valeur, on peut trouver des formules comme: «[...] l'imagination ne suit pas toujours. On réclame dans une telle affaire plus de gags originaux et moins de péripéties sans réelles surprises. Et surtout plus de provocation [...] Malheureusement on reste sur 
sa faim. " On retrouve la même tiédeur, assortie peut-être de plus de nuances, dans le quotidien Le Monde. Encore que l'infériorité numérique de termes négatifs (on trouve tout de même les piques suivantes "gags faciles" et "quelques accès de langueur ") par rapport à la recension du Parisien libéré soit compensée par le seul fait que la brièveté de l'entrefilet signifie, en soi, que le journal n'accorde pas d'importance culturelle au film (Le Monde, en tant que journal dont les lecteurs ont un capital culturel élevé n'hésite pas à consacrer une pleine page à des œuvres méritant, à son avis, l'attention de ses lecteurs).

Il existe une deuxième catégorie de journaux qui ont parlé du film de Jean-Marie Poiré et Christian Clavier (coscénariste et acteur principal) avant de connaître le succès du film, ce sont les mensuels de cinéma, non pas les mensuels que l'on pourrait dire de tradition cinéphilique (Les Cahiers du cinéma, Jeune cinéma, Positif, Le Mensuel de cinéma), mais ceux que l'on appellera "les magazines ${ }^{6}$ ", dont on dira, pour être bref, qu'ils visent le plus grand public possible (d'ailleurs les tirages de ces revues, Studio et Première, sont sans commune mesure avec les premiers). Ces deux magazines ont accordé dans leur numéro du mois de janvier une place de choix aux Visiteurs (reportage sur le tournage, interview des divers intervenants) et c'est d'eux qu'émanent les épithètes les plus laudatives (dans ce genre de magazine on dit tout de go que l'on aime le film). Le ton de la critique (qui, dans Première, se distancie, du moins de quelques pages, du reportage) est à l'encensement sans aucune restriction :

Jean-Marie Poiré et Christian Clavier, coauteurs déjà approuvés par tant de monde, frappent une nouvelle fois fort et juste. Car la vraie comédie est bien là dans sa folie redoutable et destructrice, qui parle à tous, au même instant. On a trop souvent à la bouche aujourd'hui, et pour le moindre soupçon de drôlerie, ce mot de comédie qui justifie toutes les demi-teintes et demiportions du rire [...]. Le zygomatique devient un muscle réflexe. Et instantanément moral. C'est connu, le gag réalise pour nous - et avec plus de vigueur tous les interdits que notre inconscient refoule. Et plus ça va vite, plus on aime, plus on est reconnaissant à Poiré et à ses acteurs de leur timing frénétique. [...] 
Ainsi leur duo (Jean Réno et Christian Clavier) s'impose-t-il en bienfait pour l'humanité'.

Si une large citation vient d'être faite de cet article, c'est évidemment parce qu'elle est très symptomatique de l'habitus de son auteur et, par là même, de la position de cette revue dans le champ cinématographique, intériorisée par le critique. À le lire, il ne se fait aucun doute quant à la réponse qu'il donne à la question fondamentale qui vient d'être évoquée: "Le public a-t-il raison?" D'où la répétition de formules qui placent le tandem Clavier-Poiré au rang des "créateurs" hautement estimables puisqu'ils ont déjà "été approuvés par tant de monde" et qu'ils produisent un comique "qui parle à tous». On ne peut dire plus nettement, mais sans doute inconsciemment, qu'on est inféodé au verdict du public. D'ailleurs, la position adverse dans le champ cinématographique, incarnée par ceux qui méprisent le comique de "grosse farce" est visée par l'auteur avec sa phrase: "On a trop souvent à la bouche $[\ldots]$ ". C'est là une constance des champs artistiques: le discours célébrant une œuvre ne s'élabore jamais sans égratigner les œuvres diamétralement opposées dans le champ ${ }^{8}$. Comme si le critique ne se jugeait habilité à faire l'éloge d'un pur produit du toilet-bowl humor' qu'en attaquant le comique plus "raffiné ${ }^{10}$ " (ce qu'il désigne par l'expression de comique "en demi-teinte»). Cette attitude du journaliste permet de manifester une des particularités des champs artistiques français: y règne le goût que Bourdieu appelle "distingué " et qui se caractérise par le fait que les besoins corporels fondamentaux (sexuels, excrétionnels) ne peuvent être évoqués que s'ils sont soumis "[...] à l'épuration, au raffinement, à la sublimation" (1979, p. 197). Ce goût est dans une position si dominante, et ce depuis tant d'années que lorsqu'un critique veut encenser une œuvre dont il voit bien qu'elle ressortit au goût antagoniste, "vulgaire" (ou tout autre terme, qui de toute façon sera péjoratif parce que c'est toujours le goût supérieur qui est en position de distribuer les appellations pour désigner et dénoncer le goût opposé), il s'évertue à donner bonne conscience "instantanément morale" à ses lecteurs en lançant des piques sur les œuvres qui veulent concilier humour et mise à distance du vulgaire. L'ironie, qui souligne bien à quel point nul 
ne peut échapper à la domination du goût légitime, c'est que le critique est obligé, pour justifier que l'on rie de "plaisanteries vulgaires", de mobiliser un discours savant emprunté à la psychanalyse" ("les interdits que notre inconscient refoule").

\section{Les critiques publiées dans les hebdomadaires}

Quand on se penche sur des hebdomadaires généralistes qui n'avaient pas publié de "papiers" sur ce film la semaine où il est sorti (comme Le Nouvel Observateur) ou des articulets si courts qu'ils en deviennent inconsistants, l'on constate qu'il est évoqué, non plus en tant que film, mais en tant que phénomène de société. C'est ainsi que $V . S$. D. et Le Nouvel Observateur ont publié des articles de quelques pages, l'un 10 jours, l'autre 6 semaines après sa sortie, traitant moins du film en lui-même que de son triomphe. Celui-ci fut très vite considéré comme un phénomène symptomatique d'un état d'esprit de la société (on sait que les hebdomadaires sont très friands des "phénomènes de société»). L'analyse se réduit pour l'essentiel au raisonnement suivant: les Français sont si nombreux à rire en voyant cette comédie parce que la France est "[...] morose et gagnée par le vague à l'âme" et que "[...] le rire prospère dans les crises ${ }^{12}$ ". Affirmation reposant sur un syllogisme: 1) (prémisse majeure) la France est triste; 2) (prémisse mineure) les Français ont besoin d'oublier cette tristesse; 3) (conclusion) ils veulent rire. Gageons que si Les Visiteurs avaient été un long métrage tragique et qu'il eûe remporté le même succès, le même syllogisme, mais remanié, eût été aussi utilisé.

Les auteurs de ces articles (qui ne sont pas les spécialistes de cinéma de la revue, mais les spécialistes de la rubrique "Société "), plus longs que les recensions habituelles de films, encore que de nombreuses photos et de larges extraits de déclarations des "auteurs" du film réduisent fortement la partie vraiment rédigée par les journalistes, sont amenés, cette fois, à émettre des jugements de valeur. On retrouve le même discours que dans les revues spécialisées de cinéma comme Première, analysé plus haut:

Clavier et Poiré ne font pas dans la comédie subtile et légère pour les culs coincés. Leur comique est gaulois, 
mais particulièrement bien "gaulé». Ce n'est pas de la dentelle ni du point de croix, mais plutôt tricoté à l'épée comme une cote [sic] de mailles. Et alors? Coluche ne faisait pas non plus dans la paillette ${ }^{13}$.

L'analyse que l'on vient de faire pour l'article de Première pourrait parfaitement être reprise ici. Défense du comique "graveleux" par l'attaque du goût opposé qui se trahit dans le terme "cul coincé" et culmine dans l'apostrophe "Et alors?", qui ne peut être interprétée que comme une déculpabilisation du lecteur: vous riez d'un comique qui est gras, on ne peut pas le nier, mais n'ayez pas mauvaise conscience, parce que Coluche... L'argument d'autorité "coluchienne" est plus faible (encore que depuis sa mort, il soit comme culturellement sanctifié) que le recours à la psychanalyse de Première. Le Nouvel Observateur, dont les lecteurs ont plus de capital culturel que ceux de $V$. S. D., use, pour faire l'éloge du film, d'une argumentation plus retorse, et plus adaptée à son public: "Coup génial : en faisant s'esbaudir la France profonde, l'équipe des Visiteurs nous offre un film politique. Une "visite" dans le monde des peurs françaises, dans le “bin's” des années 90. Peut-on faire plus politique? Mais attention, chut! il ne faut rien dire. Poiré et Clavier sont des comiques ${ }^{14}$." On se croirait revenu, à lire ces lignes, aux années 70, où un critique au Nouvel Observateur ne pouvait fournir meilleure justification de sa dilection d'un film qu'en lui trouvant un contenu politique. Les journalistes du Nouvel Observateur cherchent eux aussi à déculpabiliser leur public. Cependant leur méthode est différente de celle utilisée par leurs confrères de Première et de $V . S$. D. Ils ne lancent pas de piques contre le goût antagoniste, le comique de la "demi-teinte", ils ne font pas appel à l'argument d'autorité freudienne ou coluchienne, mais ils visent tout de même implicitement certaines habitudes inhérentes au goût "distingué", telles que la hiérarchie des valeurs, qui place l'œuvre politique au-dessus du film comique. La présence de ce goût légitime est perceptible dans la phrase "Mais attention, chut! il ne faut rien dire", qui ne peut être interprétée que comme une subversion du goût dominant. Les Visiteurs ou, selon Le Nouvel Observateur, sous les pavés "gras " ${ }^{15}$ du comique, le sable fin du politique. 


\section{Les revues de cinéma de tradition cinéphile}

Dans les dernières lignes de l'article de $V . S$. D., le journaliste fait preuve d'une prémonition qui dénote soit qu'il possède un sens de l'anticipation lui permettant de deviner les retournements de position avec une maestria étonnante, soit qu'il ait détenu certaines informations et qu'il en ait profité pour jouer au devin sociologique. Il termine son article par: "D'ici à ce que Les Cahiers du cinéma nous fassent, eux aussi, une poussée de rigolade! On se marre d'avance ${ }^{16}$." Et en effet, Les Cahiers $d u$ cinéma du mois de mars 1993 ont célébré Les Visiteurs. Article élogieux d'une page et entretien avec Poiré-Clavier. Cela fit du bruit dans le Landerneau de la cinéphilie.

L'exercice était périlleux pour l'auteur de la recension des Cahiers du cinéma, qui devait ${ }^{17}$ faire un "papier" très favorable à cette œuvre tout en se maintenant au niveau d' "intellectualisme" propre à la revue française de cinéma la plus célèbre. Le critique manifeste, au début de son article, son allégeance à cette tradition intellectualiste en concoctant une lecture du film que l'on peut juger, pour le moins, alambiquée. La figure de l'Auteur dans le sens négatif que lui donnent les cahieristes à la suite de Serge Daney: "La politique des auteurs est devenue, côté commerce, un marketing obligé des effets de signature " (Daney, 1983, p. 164) — est assimilée, par le truchement de longues phrases fonctionnant sur le mode du marabout-bout-de-ficelle, au seigneur du château ("[...] dans une politique du toutà-l'auteur [...] qui n'oublie jamais [...] que le metteur en scène est roi, en tout cas grand seigneur au royaume des rigolos.[...] l'auteur-seigneur du château usurpe l'auteur-citoyen du monde $\left.{ }^{18} "\right)$. Après avoir ébloui le lecteur par une interprétation si intellectuelle d'un objet filmique si éloigné du monde intellectuel, le critique revient à des procédés plus traditionnels. C'est ainsi qu'on retrouve dans cet article l'utilisation implicite du schème de pensée sur lequel se font maintes critiques laudatives: le film X est bon parce que c'est autre chose que les films $Z$. Les films Z sont ici, les films de Jean-Jacques Annaud. Ses trois derniers opus (en 1993) y sont mentionnés, et chaque fois en tant que référence-repoussoir: 1) "mieux que L'Amant"; 2) "se transformer en Ours menaçant, contre-référence au cinéma 
d'Annaud "; 3) «vision d'un Moyen Âge sale et puant à l'opposé de celle, ornementale et fétichisée, du Nom de la rose ${ }^{19}$ ". Qui a vu ces trois longs métrages d'Annaud, peut se demander pourquoi ils ont été choisis comme films-repoussoirs permettant de mieux louanger Les Visiteurs. Ils ne relèvent pas du même genre. Le mystère s'explique si l'on jette un œil sur le classement économique des cinéastes français. Jean-Jacques Annaud est le champion (du moins l'était en 1993) du top 15 des réalisateurs français ${ }^{20}$. C'est sur ce seul axe qu'il entre en concurrence avec Jean-Marie Poiré. Ils sont les deux plus grands champions de succès commerciaux français. L'un travaillant du côté du patrimoine culturel (adaptant Umberto Eco et Marguerite Duras) ou lorgnant vers Walt Disney (L'Ours), l'autre pratiquant un genre comique, qualifié par Les Cahiers du cinéma de "vraiment ambitieux", et par Le Monde de "café-théâtre". Et comme Les Cabiers du cinéma exècrent le premier, le second défini comme étant le contraire du premier se trouve, ipso facto, aimable. Paul Valéry n'aurait pu rêver une plus belle illustration de son aphorisme "Le goût est fait de mille dégoûts" (1960, p. 476).

Toujours dans la stratégie du "c'est autre chose que», le critique des Cabiers du cinéma a recours à l'attaque en règle contre le comique de goût opposé, que Première appelait "demi-teinte " et $V . S$. D., "cul coincé". Comme il règne, depuis les articles mémorables de Pascal Bonitzer dans les années70, une tradition des appellations acronymiques, ce type de comique est dénommé "BTBM" (bon ton, bon marché). À l'instar de ce qui est pratiqué dans les deux autres journaux ayant usé de cette rhétorique, il n'est donné aucun nom de film ou de cinéaste incarnant cette "nouvelle comédie française qui voudrait faire rire à peu de frais ".

Le troisième argument permettant de vanter les mérites de la comédie de Poiré étonne de la part d'une revue comme Les Cahiers du cinéma, puisqu'il dénote une vénération de l'argent. "Si on ne nous trompe pas sur la marchandise (on peut voir l'argent réuni par la production à l'image: c'est par là qu'il est passé), on ne fait guère plus de manières pour l'endommager (les objets de valeur, comme les lustres, sont méthodiquement cassés ou arrachés [...]). Dans Les Visiteurs, on voit l'argent et le beurre ${ }^{21}$." 
Rien ne montre mieux que cette citation à quel point le champ cinématographique est un champ artistique dans lequel la logique économique et la logique spécifique sont liées l'une à l'autre d'une manière inextricable. À tel point que toute affirmation de la primauté de la logique économique sur la logique spécifique doit être nuancée. C'est ainsi que dans cet argument, prôné par Les Cahiers du cinéma, synthétisé par la formule "on voit l'argent et le beurre", il y a une sorte de réponse implicite à un des principes de la logique économique que l'on peut résumer par la phrase calembouresque "un film vaut ce qu'il a coûté". Le critique des Cahiers du cinéma semble reconnaître l'importance de l'argent (allégeance à la logique économique) sans pour autant prendre à son compte l'axiome de la logique économique puisqu'il dit: "Le film est bon non pas parce qu'il coûte cher, mais parce que l'argent y est bien dépensé " (c'està-dire "jeté par les fenêtres", car il n'est pas dans la logique des agents engagés dans les champs artistiques d'avoir de la considération pour l'argent si ce n'est pour celui qui sera dilapidé).

Les Cahiers du cinéma sont avec Positif les plus anciennes revues cinéphiliques françaises ${ }^{22}$ et celles qui se sont opposées avec le plus de violence. Très souvent, elles prennent des positions diamétralement opposées sur des films, dans des numéros paraissant en même temps, donc sans qu'aucune n'ait influencé l'autre. C'est ce qui s'est passé avec les recensions des Visiteurs. La critique de Positif est parue aussi dans le numéro de mars 1993, et elle a été presque aussi négative que celle des Cahiers du cinéma a été positive. Le film a été "expédié" dans la section de la revue consacrée aux films d'importance moindre, "de $\mathrm{A}$ à $\mathrm{Z}$ " (le choix de rédiger une véritable critique ou un articulet est à lui seul révélateur de la "ligne" de la revue) dans un texte de 180 mots environ (Les Cabiers lui ayant consacré un millier de mots pour la recension et un entretien avec Clavier-Poiré de six pages). On ne s'étonnera pas de constater que ce que Positif reproche au film rejoint ce que les laudateurs ont aimé: "On aurait bien sûr souhaité un recours plus discret à diverses flatulences et ennuis gastriques, on aurait aimé que l'essentiel des gags ne se résume pas au saccage de demeures "chic" ${ }^{23}$ ". Les critiques en désaccord voient le même film, bien qu'on puisse en plaisantant affirmer le 
contraire, puisqu'ils s'accordent à lui reconnaître les mêmes caractéristiques (humour faisant mention des fonctions psychologiques et destruction fracassante d'objets luxueux), mais ils ne leur attribuent pas la même valeur parce que celle-ci découle de leur position dans le champ. Et c'est sur ce point que la sociologie peut intervenir : au lieu de considérer, comme le fait l'opinion commune sous l'influence des agents engagés dans les luttes des champs artistiques, que cette interprétation différente est déterminée par l'idiosyncrasie de chaque critique, l'analyse sociologique montre que chaque prise de position est corrélée avec la position du critique dans le champ cinématographique.

Lorsqu'on se livre à une socio-analyse des diverses prises de position à propos d'un même objet culturel (le film), on risque de passer pour un "inquisiteur terroriste, disponible pour toutes les actions de police symbolique" (Bourdieu, 1982, p. 8). Faire la sociologie d'un champ culturel ne doit pas être le moyen de se donner à bon compte l'impression qu'on se désolidarise du groupe auquel on a appartenu (la chapelle cinéphilique) en abjurant " [...] les croyances qui sont constitutives de l'appartenance à ce groupe et [en reniant] tout lien de filiation" (1982, p. 8). Le "socio-analyste" de la critique cinématographique peut être tenté de mettre à profit son analyse pour minimiser le discours critique par l'entremise de l'objectivation que produit le discours universitaire. En d'autres termes, et pour le dire plus crûment, l'auteur de ces lignes aurait échoué dans son "projet" si le lecteur en venait à penser qu'il prétend détenir la vérité sur le film Les Visiteurs. La critique que j'aurais écrite sur Les Visiteurs, si j'avais eu à le faire, et surtout si j'avais vu le film, aurait été, à n'en pas douter, déterminée, comme n'importe quelle recension d'un produit culturel, par ma position dans le champ englobant ledit produit.

Université de Paris I

\section{NOTES}

1 Il est très difficile de se donner, en passant, pour les non-initiés, une définition du concept de champ, qui est central dans la théorie, de même qu'une définition d' "habitus" ou de "capital " parce que ces notions ne " [...] peuvent être définies qu'à 
l'intérieur du système théorique qu'elles constituent, jamais à l'état isolé " (Pierre Bourdieu et Loïc J. D. Waquant, Réponses, Paris: Seuil, 1992, p. 71). Ces notions étant interdépendantes, on ne peut en définir une sans être amené à en définir une autre. Et cela ne peut se faire en quelques lignes, sinon au moins en quelques pages.

2 En ce sens, le champ cinématographique n'est pas sans rapport avec le champ journalistique, qui est soumis à une prédominance du pôle économique sur le pôle spécifique, comme le montre Bourdieu dans "L'emprise du journalisme", Actes de la recherche en sciences sociales, $\mathrm{n}^{\circ \mathrm{B}} 101-102$ (mars 1994).

3 Positif ne pratique pas la "liste officielle des 10 meilleurs films" des rédacteurs de la revue, mais publie discrètement, chaque année dans le numéro de mars ou celui de février, la liste des 12 meilleurs films de l'année écoulée, liste personnelle de Michel Ciment, rédacteur en chef officieux de la revue.

4 Voir Le Parisien libéré (28 janvier 1993).

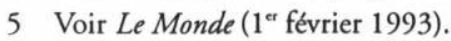

6 Sur l'opposition entre les revues spécialisées de cinéma de tradition cinéphilique et les "magazines», voir l'article de René Prédal, "France, depuis la guerre», CinémAction, $\mathrm{n}^{\circ} 69$ (1993).

7 Voir Première (janvier 1993) p. 14.

8 " "Les goûts" s'affirment de manière toute négative, par le refus opposé à d'autres goûts: en matière de goût, plus que partout, toute détermination est négative" (Pierre Bourdieu, La Distinction. Critique sociale du jugement. Paris: Minuit, 1979, p. 60).

9 C'est une formule employée à propos des Visiteurs dans un article du magazine américain Time (20 novembre 1993) lors de son compte rendu du cinquième festival du film français de Sarasota.

10 La présence constante de guillemets, chaque fois qu'il s'agira de mettre une "étiquette" sur un type de comique, n'a pas pour fonction, fût-elle inconsciente, de suggérer que je me refuse à qualifier tout type de comique, mais de rappeler qu'en matière de dénomination des types de comique, il est impossible de faire abstraction des luttes symboliques entre le goût dominant et les autres types de goûts parce que la plupart des termes qui sont à notre disposition en ce domaine ont été forgés par le goût dominant pour (dis)qualifier les autres goûts.

11 La psychanalyse a d'ailleurs tiré cette conception de l'œuvre de Kant (mais à celui-là qui n'a jamais rien pris?), qui dans Critique de la faculté de juger, véritable évangile philosophique du goût "légitime", fait de la négation de la jouissance le signe le plus sûr de la qualité d'une œuvre d'art: " [...] tout plaisir, même si des concepts éveillant des idées esthétiques en sont l'occasion, est une sensation animale, c'est-à-dire corporelle" (Euvres philosophiques, tome 2, Paris: La Pléiade, 1985, p. 1123).

12 Voir Le Nouvel Observateur (11-17 mars 1993).

13 Voir V.S. D. (4 au 10 février 1993).

14 Voir Le Nouvel Observateur (11-17 mars 1993).

15 C'est d'ailleurs l'adjectif employé par le spécialiste de cinéma de la revue: "C'est du bon rire, consistant, un poil gras, du rire qui tient au corps et aux côtes" (Alain Riou, Le Nouvel Observateur, 11-17 mars 1993). Notons que lorsque le film est sorti, le magazine n'en avait pas dit un mot et que dans ce numéro, six semaines après sa sortie, Alain Riou écrit une courte recension publiée en encart au milieu des pages consacrées à son succès.

16 Voir V.S. D. (4 au 10 février 1993).

17 L'emploi de ce terme appartenant au champ lexical de l'obligation (ainsi qu'un autre, "allégeance", quelques lignes plus loin) n’implique pas que je considère le jour- 
naliste des Cabiers du cinéma, auteur de cette recension, comme un mercenaire cynique ayant écrit cet article dans la ligne (comme on disait aux heures glorieuses du P. C.) de la revue dans la plus parfaite insincérité. Penser cela serait faire preuve d'une méconnaissance impardonnable du fonctionnement des revues de cinéma. La sincérité de l'auteur de cet article ne peut pas être mise en doute. Mais l'expérience m'a appris qu'il est très difficile pour certains d'admettre que les adeptes de la socioanalyse portent au jour l'inconscient social des agents sans douter de la sincérité de ces derniers.

18 Cahiers du cinéma, $\mathrm{n}^{\circ} 465$ (mars 1993) p. 83.

19 Idem.

20 Voir Le Film français, n 2478 (29 octobre 1993).

21 Cahiers du cinéma, $\mathrm{n}^{\circ} 465$ (mars 1993) p. 83.

22 Les Cahiers du cinéma sont nés en 1951 et Positif, en 1952; ces deux titres appartiennent donc aux deux plus anciennes revues de cinéma mensuelles n'ayant jamais changé de titre.

23 Voir Positif, n 385 (mars 1993) p. 50.

\section{OUVRAGES CITÉS}

Bourdieu, Pierre et Loïc J. D. Waquant. Réponses. Paris: Seuil, 1992.

Bourdieu, Pierre. Question de sociologie. Paris: Minuit, 1984.

Bourdieu, Pierre. Leçon sur la leçon. Paris: Minuit, 1982.

Bourdieu, Pierre. La Distinction. Critique sociale du jugement. Paris: Minuit, 1979.

Daney, Serge. La Rampe. Paris: Gallimard, 1983.

Kant, E. Critique de la faculté de juger. Paris: La Pléiade, 1985.

Valéry, Paul. «Tel Quel ». CEuvres, tome 3. Paris: La Pléiade, 1960. 